\title{
Fortalecimiento de la comprensión lectora a través del uso de las TIC en estudiantes de educación primaria ${ }^{1}$
}

\section{Strengthening of the reading comprehension across the use of the TIC in students of primary education}

DOI: http://dx.doi.org/10.17981/cultedusoc.9.3.2018.46

\author{
Artículo de investigación. Fecha de recepción: 15/06/2018. Fecha de aceptación: 27/11/2018 \\ Germán Durán²; \\ Yeritza Rozo; Alida Soto; Luz Arias y Emilse Palencia ${ }^{3}$ \\ EUM San José de Plato (Colombia) \\ germanduran01@hotmail.com
}

Para citar este artículo:

Durán, G., Rozo, Y., Soto, A., Arias, L. y Palencia, E. (2018). Fortalecimiento de la comprensión lectora a través del uso de las TIC en estudiantes de educación primaria. Cultura. Educación y Sociedad 9(3), 401-406. DOI: http://dx.doi.org/10.17981/cultedusoc.9.3.2018.46

\section{Resumen}

La lectura y escritura son procesos cognitivos básicos que hacen parte de las competencias comunicativas que deben desarrollar los estudiantes desde educación básica por lo anterior el objetivo del estudio es fortalecer la comprensión lectora mediante la utilización de herramientas tecnológicas, en pro de aumentar el rendimiento académico de los estudiantes. Se orientó bajo una metodología cualitativa con un alcance descriptivo con una población participante de treinta (30) estudiantes de la E.U.M. Sede San José. Finalmente se observó que la compresión lectora de los estudiantes aumento considerablemente gracias a la utilización de herramientas tecnológicas como estrategia de aplicación potenciando sus competencias lo que permitió mejorar el rendimiento académico no solo en Lengua Castellana; sino también en las distintas áreas vistas.

Palabras clave: Comprensión, desempeño, influencia, lectura, tecnología.

\section{Abstract}

The reading and writing are cognitive basic processes that do part of the communicative competitions that the students must develop from basic education for previous the aim of the study it is to strengthen the reading comprehension by means of the utilization of technological tools, in favor of increasing the academic performance of the students. There was orientated under a qualitative methodology by a descriptive scope by a population participant of thirty (30) students of the E.U.M. Headquarters San Jose. Finally was observed that I increase the reading compression of the students considerably thanks to the utilization of technological tools as strategy of application promoting his competitions what allowed to improve the academic not alone performance in Castilian Language; but also in the different seen areas.

Keywords: Comprehension, performance, influence, reading, technology.

1 Este artículo ha sido derivado del Programa de Fortalecimiento de la Cultura Ciudadana y Democrática CT+I a través de la IEP apoyada en TIC en el Departamento de Magdalena: CICLON

2 Líder del grupo de investigación "Redes Docentes San José", de la EUM San José de Plato.

3 Docentes del grupo de investigación "Redes Docentes San José", de la EUM San José de Plato.

- The author; licensee Universidad de la Costa - CUC.

Cultura, Educación y Sociedad vol. 9 no. 3, pp. 401-406. Diciembre, 2018

Barranquilla. ISSN 2389-7724 Online 


\section{Introducción}

El proceso de comprensión lectora implica la interpretación de los significados de un texto escrito, de manera activa y constructiva. En él se colocan en interjuego habilidades y estrategias mentales para el procesamiento de la información que se recibe, así como para la construcción de los significados del texto y producción de nueva información a partir de los análisis realizados y la informació previa que posee el autor (Ramos, 2006).

Investigadores de las ciencias de la Educación, han concentrado sus esfuerzos en las búsquedas teóricas que expliquen la finalidad de la comprensión lectora, teniendo en cuenta que se trata de un proceso de elaboración que implica mucho más que integrar significados obtenidos de una cadena de palabras decodificada con anterioridad (Noriega, 1998). Los procesos de lectura pautados en la actualidad, en los cuales se promueve la calidad ortográfica, hacen una invitación muy cordial a cuestionarse acerca de que es leer bien, y por ende a entender de qué es lo que se espera de un buen lector (Jaramillo, Montaña, Reina, 2006).

La dimensión cognitiva desde la que se aborda la comprensión lectora, establece que existen diversos niveles desde donde se procesa la información, que se establecen desde los más básicos (en los que se captas grafemas, reconocimiento de palabras, funciones a las palabras que configuran determinadas oraciones y decodificación grafema-fonema (Canet, Richards, Burin, 2009).

Específicamente, en los procesos de enseñanza-aprendizaje, es indispensable, desde el punto de vista psicológico, la competencia lectora. La razón de ello, es que es un proceso que determina el éxito o fracaso en las demás áreas académicas, puesto que es necesaria para la comprensión de los contenidos asumidos en ellas, de manera que si una persona no tiene las competencias suficientes para realizar la comprensión de textos, esto afectaría directamente al resto de las áreas curriculares (Gutiérrez, Pérez, 2012).

Algunos autores han concentrado sus esfuerzos en el uso de textos argumentativos, como herramienta para el mejoramiento de la comprensión lectora, puesto que consideran que la tipología textual es poco conocida tanto por docentes, como por los estudiantes. De manera contraria, los estudios indican que los textos narrativos son los más empleados, incluso en la educación superior (Caballero, 2008).

La mayoría de los niños con dificultades en la comprensión lectora poseen rendimientos inferiores en un conjunto de procesos cognitivos, entonces establecer que un perfil bajo en rendimiento en comprensión lectora está directamente relacionado a el bajo rendimiento académico de los estudiantes en sus demás asignaturas, es de mucho sentido, debido a que una cosa influye a la otra (Canet, Burin, Urquijo, 2013).

La intención del lector, cuando realiza la lectura, influye en su captación, durante el proceso lector se llevan a cabo actividades diversas, tales como: revisar un texto para captar la idea general, leer en profundidad para conocer detalladamente un texto, leer por placer y trasladarnos a diferentes escenarios, leer para argumentar y asumir juicios críticos, leer para aprender y memorizar, entre otros fines (González, 2012).

Desde la perspectiva de la evaluación por competencias, no sólo es necesario saber si una persona sabe leer o cuánto lee, sino que además es necesario identificar si es capaz de identificar ideas y argumentos en un texto, reconoce problemas y diversidad de posturas de los autores (Suarez, Moreno, Godoy, 2010). En este sentido, se concibe a un individuo activo que interpreta un texto en función de su experiencia, necesidades y conocimientos, y al mismo tiempo, evalua, comprende, desecha y selecciona aquello que considera importante (Arteaga, 2001). 
La actualidad tecnológica ha traido consigo procesos educativos más personalizados, con poco acopañamiento familiar y desarrollo de competencias lectoras débil, razón por la que se plantea la presente investigación (Gómez, 2011).

Las exigencias de la Sociedad del Siglo XXI traen consigo cambios en la educación, que requieren sin duda de personas creativas, emprendedoras, visionarias, con amplias habilidades y conocimientos en tecnología, para garantizar un aprendizaje "a lo largo de la vida" (Maldonado, Sanjuanelo, Clavijo, 2012). Sin embargo, tal aprendizaje debe realziarse de una manera cooperativa, con el fin de generar estructuras que den cuenta de una interdependencia socail con todas las variables que las regulan, lo cual se realizaría de manera más efectiva con el uso de la tecnología. En este sentido, se identifica la utilidad y positiva contribución de la tecnología y el aprendizaje cooperativo en el desarrollo de competencias de comprensión de lectura (Valdebenito y Duran, 2013).

En una investigación sobre la actitud de los estudiantes de secundaria frente a la tecnología informática, lso resultados indican que la mayoría de los estudiantes consideran que la lectura frente al computador se realiza de manera más fácil, puesto que al presentar menos información al mismo tiempo, hace que el lector se concentre mucho más y no pierda de vista tan fácilmente el lugar por el que va la lectura (Henao, 2006).

Por lo anterior se plantea que el objetivo del presente estudio corresponde a fortalecer los niveles de comprensión lectora en los niñas y niñas de los grados cuarto y quinto de la institución intervenida, a través de la implementación de las TIC como estrategia pedagógica teniendo como intención mejorar el rendimiento académico de los estudiantes.

\section{Metodología}

El abordaje metodológico del presente estudio se realizara desde un enfoque cualitativo con un alcance descriptivo, implementando un diseño de investigación de acción participación; teniendo en cuenta que se busca transformar una realidad a partir del estudio de una problemática (Hernández, 2014).

\section{Participantes}

En cuanto a la población beneficiada con el estudio, se tomó una muestra representativa de treinta (30) estudiantes de la E.U.M. Sede San José, distribuidos en cuarto y quinto grado, con edades que oscilaron entre los once (11) y doce (12) años.

\section{Técnicas e Instrumentos}

Como técnica de recolección de información se elige la observación participante, debido a que el investigador tiene la facultad de participar, escuchar y por ende observar el fenómeno en un contexto real (Hernández, 2014). En cuanto a los instrumentos se selecciona el diario de campo para llevar el registro de la información más significativa.

\section{Procedimiento}

La población participante del estudio fue inicialmente clasificada en tres (3) grupos de 10 estudiantes, grupo A, B, y C en cada uno de ellos se llevará a cabo la aplicación de talleres por medio de los cuales se identificara cual es el grado de comprensión lectora que manejan, y posteriormente se describirán los aspectos que se encuentren en el proceso.

En los talleres se les pedirá a los estudiantes que desarrollen lecturas de manera crítica y que elaboren actividades 
con preguntas relacionadas a la temática trabajada, estos contenidos son proyectados a través del uso de computadores y tabletas disponibles en la institución, ya que se implementan las tecnologías de la información y comunicación como herramienta de trabajo.

\section{Resultados}

A continuación se muestran los resultados encontrados en la aplicación de las actividades diseñadas para el estudio, los hallazgos son los siguientes:

TABLA 1

\begin{tabular}{cc}
\hline Sesión & Actividad \\
\hline & \\
& \\
Sesión 1 & Taller sobre el uso de los \\
dispositivos tecnológicos
\end{tabular}
dispositivos tecnológicos

Sesión 2

Sesión 3
Taller de lectura critica

Taller de lectura critica
Resultado

Durante la aplicación de la actividad los estudiantes asistieron al salón múltiple de la institución, en este una parte de los estudiantes tomo computadores y la otra tablets, los docentes le explicaron como buscar la lectura seleccionada para la actividad. En primera instancia se les capacito para el uso de los aparatos tecnológicos, debido a que algunos de ellos no manejan de manera adecuada el sistema, se mostraron motivados e interesados en aprender teniendo en cuenta que estaban utilizando el salón equipado con los dispositivos electrónicos como estrategia pedagógica.

En el presente taller se inició con la explicación de los docentes frente a que se desarrollaría durante la sesión, por lo tanto los maestros expresaron que se buscaría el texto "la bobina maravillosa" y a partir de su lectura se responderían una serie de preguntas relacionadas a esta. En el transcurso de la actividad los estudiantes evidenciaron motivación por la lectura que se les asigno, debido que al final realizaban preguntas y respondían en su mayoría de manera acertada a las preguntas formuladas por el docente.

En esta actividad los estudiantes llevaron a cabo otra sesión de lectura, pero con la diferencia de que las preguntas no fueron formuladas de manera oral por el docente sino; que se les entrego un cuadernillo virtual con los interrogantes e ilustraciones relacionadas a la lectura. En este espacio los estudiantes se mostraron bastante animados a participar en la actividad, manifestando que "profe, de esta manera si me ha gustado leer no están aburrido como en el salón de clase", "seño, es muy chévere leer en el computador y que uno pueda ver dibujos", "profe, el así es más bacano porque en los libros uno se aburre ve es pura letra, además usamos el computador o la Tablet”. Resolviendo las preguntas de la lectura "la bruja peluja" sin identificarse actitudes de aburrimiento o resistencia.

Fuente: elaboración propia. 
A partir de las actividades realizadas y descritas en la tabla anterior, los docentes procedieron a revisar detalladamente las respuestas a las preguntas de las lecturas aplicadas para así determinar el nivel de compresión lectora que tienen los niños y niñas participantes del estudio, cabe resaltar que la población fue dividida en cuatro grupos lo que corresponde al 100\% de la muestra por lo tanto se plantea que; el grupo A reflejo que no cuentan con unas competencias lectoras afianzadas ya que al implementarse los talleres los estudiantes no respondieron las preguntas de manera correcta en su mayoría lo que denota baja comprensión en la lectura y la poca frecuencia en la que se realiza el ejercicio.

En cuanto al grupo B, los estudiantes mostraron un nivel de comprensión lectora mayor al grupo anterior, por la razón de que al evaluar las respuestas en cuanto al contenido de esta y si era acertada o no; el grupo de 10 estudiantes posee mejores habilidades ya que se evidenciaron respuestas más elaboradas y más cantidad de aciertos, lo que presume que al impartir las actividades de lectura crítica los estudiantes mejoran sus competencias lectoras. Referente al grupo C, la comprensión lectora es superior, porque los estudiantes lograron responder en las dos aplicaciones de las lecturas las preguntas correctamente y con un contenido denso y coherente a lo formulado, sin embargo los maestros identificaron que el trabajo debe ser constante para el mejoramiento permanente de la competencia.

\section{Discusión}

Desde la implementación de las actividades diseñadas para el estudio los maestros identificaron que es de vital importancia que con los estudiantes se implementen estratégicas pedagógicas que permitan el desarrollo de la comprensión lectora en la comunidad estudiantil, considerando las dificultades que se presentan entre ellos y en este sentido que sean coherentes al nivel de competencias que posean.
En este orden fomentar la lectura comprensiva y critica en los estudiantes desde la básica primaria es de gran relevancia teniendo en cuenta que el proceso de enseñanza de la Lengua Castellana consta en su mayoría del ejercicio de leer de manera constante, beneficiando su aprendizaje y desenvolvimiento en las siguientes etapas escolares. Asimismo mantener el uso de las TIC en el proceso de enseñanza-aprendizaje, ya que es una estrategia metodológica que predice éxito en cuanto a que motiva y genera interés por el uso de los aparatos tecnológicos.

Finalmente se pretende que el proyecto se implemente en toda la comunidad estudiantil en general, para originar espacios donde el estudiante visualice la lectura como un componente fundamental en su formación y modificar desde el área administrativa los planes curriculares impartiendo la investigación como una metodología de enseñanza que complemente el proceso de aprendizaje.

\section{Referencias}

Caballero, E. (2008). Comprensión lectora de los textos argumentativos en los niños de poblaciones vulnerables escolarizados en quinto grado de educación básica primaria. [Tesis maestría]. Universidad de Antioquia, Medellín.

Canet-Juric, L., Urquijo, S., Richard's, M. M. y Burin, D. (2009). Predictores cognitivos de niveles de comprensión lectora mediante análisis discriminante. International Journal of Psychological Research, 2(2).

Colomer, T. (1997). La enseñanza y el aprendizaje de la comprensión lectora. Revista Signos, 20, 6-15.

Gómez, J. (2011). Comprensión lectora y rendimiento escolar: una ruta para mejorar la comunicación. COMUNI@ CCIÓN: Revista de Investigación en Comunicación y Desarrollo, 2(2). 
Gonzales, M. (2012). Comprensión lectora. Intención o propósito del lector. Capítulo 3. Obtenido de http://www. mailxmail.com/curso-comprension/ comprension-lectora-intencion-proposito-lector

Guerrero, H., Crissien, T. y Paniagua, R. (2017). Proyectos Educativos Institucionales Colombianos (PEI): Educación Inclusiva a través de la autoevaluación. Opción, 33(84). 218266.

Gutiérrez-Braojos, C. y Pérez, H. S. (2012). Estrategias de comprensión lectora: enseñanza y evaluación en educación primaria. Profesorado. Revista de Currículum y Formación de Profesorado, 16(1). 183-202.

Henao, O. (2006). Evidencias de la investigación sobre el impacto de las tecnologías de información y comunicación en la enseñanza de la lecto-escritura. Revista Educación y Pedagogía, XVIII(44). 71-87.

Hernández, R. (2014). Metodologia de la investigación. México, D.F.: Mc Graw Hill.

Jaramillo, A., Montaña, G., Reina, R. y Marcela, L. (2006). Detección de errores en el proceso metacognitivo de monitoreo de la comprensión lectora en niños. Revista Latinoamericana de Ciencias Sociales, Niñez y Juventud, 4(2), 75-95.

Maldonado, A., Sanjuanelo, M. y Clavijo, J. (2011). Potenciar la comprensión lectora desde la tecnología de la información. Escenarios, 9(2). 26-36.

Martinez, J. y Garcia, L. (2012). Promoción de la Salud. España: Paraninfo.
Matera, O., Ariza, H., Angulo, D. y Vargas, R. (2015). Influencia de la comprensión lectora primaria, año 2015. Obtenido de https:// es.scribd.com/document/269824521/2

Monroy, J. y Gómez, B. (2009). Comprensión lectora. Revista Mexicana de Orientación Educativa, 6(16), 37-42.

Noriega, E. (1998). Estudio cualitativo de los niveles de comprensión lectora de un grupo de niños deficientes y buenos lectores antes y después de un programa de intervención. Psicología, III(3): 159-71.

OCDE. (s.f.). El progrma PISA de la OCDE. París: OCDE. Obtenido de https://www. oecd.org/pisa/39730818.pdf

Pinto, M. y Misas, M. (2014). La educación inicial y la educación preescolar: Perspectivas de desarrollo en colombia y su importancia en la configuración del mundo de los niños. Cultura Educación y Sociedad 5(1), 119-140.

Ramos, C. (2006). Elaboración de un instrumento para medir comprensión lectora en niños de octavo año básico. Onomázein, 2(14). 197-210.

Suárez, A., Moreno, J. y Godoy, M. J. (2010). Vocabulario y comprensión lectora: algo más que causa y efecto. Álabe, 1(1). http:// dx.doi.org/10.15645/Alabe.2010.1.7

Truyoth, E. y Villalobos, A. (2012). Etnografia. Lima: Universidad de Córdoba.

Valdebenito, V. y Duran, D. (2013). La tutoría entre iguales como un potente recurso de aprendizaje entre alumnos: efectos, fluidez y comprensión lectora. Perspectiva Educacional, Formación de Profesores, $52(2)$.

Vallés, A. (2005). Comprensión lectora y procesos psicológicos. Liberabit, 11(11), $41-48$ 